\title{
The independent patient factors that affect length of stay following hip fractures
}

\author{
T Richards ${ }^{1}$, A Glendenning ${ }^{2}$, D Benson ${ }^{1}$, S Alexander ${ }^{3}$, S Thati ${ }^{1}$ \\ ${ }^{1}$ Trauma and Orthopaedic Department, Ysbyty Gwynedd, Bangor, Wales, UK \\ ${ }^{2}$ Swansea University Medical School, Swansea, Wales, UK \\ ${ }^{3}$ Orthogeriatrics Department, Ysbyty Gwynedd, Bangor, Wales, UK
}

\section{ABSTRACT}

INTRODUCTION Management of hip fractures has evolved over recent years to drive better outcomes including length of hospital stay. We aimed to identify and quantify the effect that patient factors influence acute hospital and total health service length of stay.

METHODS A retrospective observational study based on National Hip Fracture Database data was conducted from 1 January 2014 to 31 December 2015. A multiple regression analysis of 330 patients was carried out to determine independent factors that affect acute hospital and total hospital length of stay.

RESULTS American Society of Anesthesiologists (ASA) grade 3 or above, Abbreviated Mental Test Score (AMTS) less than 8 and poor mobility status were independent factors, significantly increasing length of hospital stay in our population. Acute hospital length of stay can be predicted as 8.9 days longer when AMTS less than 8, 4.2 days longer when ASA grade was 3 or above and 20.4 days longer when unable to mobilise unaided (compared with independently mobile individuals). Other factors including total hip replacement compared with hemiarthroplasty did not independently affect length of stay.

CONCLUSIONS Our analysis in a representative and generalisable population illustrates the importance of identifying these three patient characteristics in hip fracture patients. When recognised and targeted with orthogeriatric support, the length of hospital stay for these patients can be reduced and overall hip fracture care improved. Screening on admission for ASA grade, AMTS and mobility status allows prediction of length of stay and tailoring of care to match needs.

\section{KEYWORDS}

Hip fracture - Neck of femur - Length of stay

Accepted 18 February 2018

CORRESPONDENCE TO

Tomos Richards, E: tomosrichards@doctors.org.uk

\section{Introduction}

Hip fractures are a common injury in the elderly, which poses a huge burden on health services worldwide. Hip fracture care costs over $£ 1.1$ billion each year to the health and social services in the UK and necessitates input from a wide range of departments and agencies. ${ }^{1}$ With an ageing population, the UK incidence of hip fractures is expected to increase from 79,000 to 104,000 per year by 2025 and this burden of care will also multiply to an estimated yearly cost of $£ 1.5$ billion. ${ }^{1}$ Worldwide, this cost is also increasing and is predicted to reach US\$25.3 billion in the United States by $2025 .^{2}$ These patients currently occupy over 1.5 million bed days each year in the NHS. ${ }^{3}$ Length of hospital stay varies across the UK, with an overall mean total of 20.2 days, of which 15.9 days is in the acute hospital. ${ }^{3}$ The acute length of stay corresponds to the time to discharge in the acute treating hospital and total length of stay corresponds to total time spent in NHS care from admission to the acute treating hospital, including time spent in rehabilitation hospitals until discharge to their own home or care facility. ${ }^{3}$ The National Hip Fracture Database recognises that there is some underreporting of community and rehabilitation stays in England, which may influence its lower total length of stay compared with other areas of the UK. ${ }^{3}$ The acute length of stay is higher in areas such as Wales with an overall mean of 19.6 days, ${ }^{3}$ while outside the UK acute length of stay is as low as 9.8 days in Finland ${ }^{4}$ and 6.9 days in some centres in the USA. ${ }^{5}$ Patient mortality rates after discharge have been shown to increase with length of stay of stay in some units, ${ }^{6}$ with the converse being true in length of stay under 10 days in a Swedish study. ${ }^{7}$ There are many factors that affect the overall length of stay, both individual patient factors and those concerning design of health and social services. If these factors are identified and targeted, better patient satisfaction may be achieved in this patient group, as well as reducing overall costs and improving availability of acute beds for the health service. We aim to identify and quantify these patient factors associated with longer total length of stay in 
a cohort of patients in a district general hospital within Wales, an area of higher than average length of stay within the UK. This information can be used to further plan services and patient care and make improvements to reduce length of stay and therefore cost and bed pressures.

\section{Methods}

A retrospective cohort study of all hip fracture patients treated in a single district general hospital in North Wales over a two-year period from 1 January 2014 to 31 December $2015(n=663)$. The primary measures were acute hospital and total health service inpatient length of stay with identification and quantification of factors that independently affect them. Data were collected from the National Hip Fracture Database, including demographics, fracture pattern, surgery performed, place of residence prior to admission, mobility prior to admission, Abbreviated Mental Test Score (AMTS) and American Society for Anesthesiologists (ASA) grade. Patients who died prior to discharge $(n=63)$ were excluded from the analysis, as were patients with incomplete data $(n=268)$ and patients with simultaneous bilateral fractures (two records, one patient) leaving 330 patients for final analysis. Length of stay in the acute hospital and total NHS stay were compared between individual factors using independent t-tests for dichotomous variables and analysis of variance for other categorical variables with more than two levels. A multiple regression model was then used to determine the adjusted effect of each individual factor and factors with $P$-value less than 0.05 were considered statistically significant. A separate regression model was used to determine the effect of new placement on length of stay as this is a variable derived from the pre-fracture residence and final discharge destination data points within our model. Analyses were carried out using Microsoft Excel (version 14) and IBM SPSS (version 22) statistical software. AMTS were grouped into normal $(\geq 8)$ or abnormal $(\leq 7)$ for analysis. ${ }^{8}$

\section{Results}

The overall mean length of stay in the acute hospital was 15.6 days. The mean total NHS length of stay was 35.6 days. The mean age of the sample was 82.2 years (range 56-101 years) with 259 (78\%) being female. Inpatient mortality rate was 63 from $662(9.5 \%)$ (not surviving to discharge). Demographics of the sample are illustrated in Table 1. Patients admitted from their own home, discharged to their own home and undergoing total hip replacement (THR) had a significantly shorter acute and total length of stay. Patients aged less than 80 years also had a shorter total length of stay. When these factors were adjusted for the confounding effects of the other associated variables through the multiple regression model, they did not reach statistical significance. There was no correlation between time to orthogeriatrician review and total or acute length of stay $(P=0.83$ and $P=0.406$, respectively). There was also no difference in total or acute length of stay between patients reviewed preoperatively by an orthogeriatrician or not $(P=0.599$ and $P=0.705$, respectively). There was no correlation between time from admission to surgery and acute or total length of stay ( $P=0.114$ and $P=0.564$, respectively). Only three patient factors were identified in our model as independent predictors of increased length of stay: reduced pre-fracture mobility, ASA score 3 or more and AMTS less than 8 . All three were independent factors associated with increased acute length of stay and low AMTS and reduced prefracture mobility with total length of stay. Table 2 shows the adjusted length of stay change for individual patient factors determined by our regression model. New placement in a residential or nursing home had no effect on acute length of $\operatorname{stay}(P=0.095)$ but a statistically significant effect on total length of $\operatorname{stay}(P=0.000)$ when adjusted for significant patient factors of ASA grade, AMTS and prefracture mobility. This equated to a predicted increase in adjusted length of stay of 22.5 days when compared with no new placement.

\section{Discussion}

There is a huge worldwide variation in length of stay in both the acute hospital and peripheral rehabilitation units for hip fracture patients. A large amount of both intrinsic patient-related and extrinsic system-related factors govern the length of stay of an individual patient. With the ageing population, it is vital to optimise this length of stay for the patient's quality of life and to reduce the burden on health and social services. Recognition of these patient factors enables service planning and interventions to optimise the care of these individuals.

Our study population was similar in demographics to worldwide hip fracture patients in terms of age, sex and fracture pattern, indicating that the findings are largely transferrable to populations across the developed world. ${ }^{3,9,10}$ The setting, a district general hospital, means that services are similar to many non-specialised centres throughout the UK and the rest of the developed world.

There was a statistically significant increase in length of stay of 8.9 days (acute) and 15.3 days (total health service) in patients with an AMTS of less than 8, which is consistent with other literature. ${ }^{11}$ Postoperative complication rates such as infection, ${ }^{12}$ pulmonary embolism ${ }^{13}$ and dislocation $^{14}$ are higher in these patients, which may contribute to this difference. Dementia increases the risk of sustaining ${ }^{15}$ and the cost of managing ${ }^{16}$ hip fractures and therefore additional care and input is required in this subgroup. Hip fracture patients with dementia should be identified early with AMTS screening on admission, as their care needs will be greater. Early involvement of orthogeriatricians to medically optimise, perform falls assessments and coordinate care and rehabilitation needs is vital in these patients. ${ }^{17}$ Compliance with rehabilitation is more difficult in this group but, with intensive programmes, comparable outcomes to cognitively intact patients may be achieved. ${ }^{18}$

The preoperative mobility status of the patients had a strong independent significant effect on the length of stay. The acute hospital length of stay of a patient unable to 


\begin{tabular}{|c|c|c|c|c|c|}
\hline Patient factor & Prevalence (\%) & Length of acute stay (days) & $P$-value & Total length of stay (days) & $P$-value \\
\hline \multicolumn{6}{|l|}{ Age (years): } \\
\hline$<80$ & 37.0 & 13.9 & & 27.9 & \\
\hline $80-90$ & 48.5 & 16.8 & & 39.4 & \\
\hline$>90$ & 14.5 & 16.3 & 0.336 & 42.6 & 0.04 \\
\hline \multicolumn{6}{|l|}{ Sex: } \\
\hline Male & 22.1 & 16.7 & & 36.1 & \\
\hline Female & 77.9 & 15.3 & 0.531 & 33.5 & 0.554 \\
\hline \multicolumn{6}{|l|}{ Side: } \\
\hline Left & 48.5 & 15.4 & & 36.9 & \\
\hline Right & 51.5 & 15.9 & 0.788 & 36.3 & 0.702 \\
\hline \multicolumn{6}{|l|}{ AMTS: } \\
\hline Normal & 70.6 & 12.5 & & 23.6 & \\
\hline Low & 29.4 & 23.1 & 0.000 & 49.5 & 0.000 \\
\hline \multicolumn{6}{|l|}{ Residence: } \\
\hline Own home & 78.5 & 13.7 & & 27.8 & \\
\hline Other & 21.5 & 22.6 & 0.007 & 38.4 & 0.022 \\
\hline \multicolumn{6}{|l|}{ ASA grade: } \\
\hline $1-2$ & 42.1 & 11.7 & & 27.5 & \\
\hline $3-5$ & 57.9 & 18.5 & 0.000 & 41.5 & 0.000 \\
\hline \multicolumn{6}{|l|}{ Mobility: } \\
\hline Unaided & 43.9 & 11.6 & & 24.6 & \\
\hline 1 stick & 21.0 & 16.7 & & 40.4 & \\
\hline 2 sticks or frame & 32.0 & 18.5 & & 44.7 & \\
\hline Unable to mobilise unaided & 3.1 & 36.9 & 0.000 & 63.6 & 0.000 \\
\hline \multicolumn{6}{|l|}{ Discharge to: } \\
\hline Own home & 71.8 & 13.4 & & 32.0 & \\
\hline Other & 28.2 & 23.5 & 0.002 & 47.0 & 0.002 \\
\hline \multicolumn{6}{|l|}{ Fracture type: } \\
\hline Intertrochanteric & 37.3 & 14.4 & & 36.5 & \\
\hline Intracapsular, displaced & 49.7 & 16.2 & & 34.4 & \\
\hline Intracapsular, undisplaced & 6.7 & 20.3 & & 32.4 & \\
\hline Subtrochanteric & 6.4 & 13.7 & 0.388 & 43.9 & 0.635 \\
\hline \multicolumn{6}{|l|}{ Operation: } \\
\hline Total hip replacement & 9.5 & 9.7 & & 15.7 & \\
\hline Other & 90.5 & 16.3 & 0.000 & 31.3 & 0.000 \\
\hline
\end{tabular}

mobilise unaided with a stick or frame was 20.4 days greater than patients mobilising unaided. It is known that mobility is usually decreased after hip fracture surgery, however this can be optimised with intensive physiotherapy and rehabilitation. ${ }^{19}$ Mobility is also inversely correlated with mortality after surgery. ${ }^{20}$ Patients who are independently mobile can be expected to be discharged sooner; however, early rehabilitation is essential in improving functional outcomes for all patients. ${ }^{21}$

An ASA grade of 3 or more was associated with greater total length of stay of 4.2 days compared with ASA grade 2 or more. We grouped the ASA gradings into 3 or more and 
Table 2 Adjusted additional length of stay for individual factors.

\begin{tabular}{|c|c|c|c|c|}
\hline Patient factor & Added acute length of stay (days) & $P$-value & Aded total length of stay (days) & $P$-value \\
\hline Baseline length of stay & $7.234(1.441,13.027)$ & & & \\
\hline \multicolumn{5}{|l|}{ Age (years): } \\
\hline$<80$ & referent & & referent & \\
\hline $80-90$ & $-0.259(-4.204,3.687)$ & 0.898 & $5.581(-2.164,13.327)$ & 0.158 \\
\hline$>90$ & $-3.739(-9.445,1.968)$ & 0.199 & $2.443(-8.728,13.615)$ & 0.668 \\
\hline \multicolumn{5}{|l|}{ Sex: } \\
\hline Male & referent & & referent & \\
\hline Female & & 0.439 & $1.370(-7.071,9.811)$ & 0.750 \\
\hline Side: & $1.677(-2.567,5.922)$ & & & \\
\hline Left & referent & & referent & \\
\hline Right & $.808(-2.658,4.274)$ & 0.648 & $-.315(-7.119,6.489)$ & 0.928 \\
\hline \multicolumn{5}{|l|}{ AMTS: } \\
\hline Normal & referent & & referent & \\
\hline Low & $8.893(4.173,13.613)$ & 0.000 & $15.278(6.014,24.542)$ & 0.001 \\
\hline \multicolumn{5}{|l|}{ Residence: } \\
\hline Own home & referent & & referent & \\
\hline Other & $1.129(-4.141,6.398)$ & 0.675 & $-8.431(-19.473,2.611)$ & 0.022 \\
\hline \multicolumn{5}{|l|}{ ASA grade: } \\
\hline $1-2$ & referent & & referent & \\
\hline $3-5$ & $4.180(.375,7.985)$ & 0.031 & $6.208(-1.310,13.727)$ & 0.106 \\
\hline \multicolumn{5}{|l|}{ Mobility: } \\
\hline Unaided & referent & & referent & \\
\hline 1 stick & $6.041(1.422,10.661)$ & 0.010 & $13.768(4.710,22.825)$ & 0.003 \\
\hline 2 sticks or frame & $3.742(-.713,8.197)$ & 0.100 & $11.942(3.247,20.638)$ & 0.007 \\
\hline Unable to mobilise unaided & $20.435(10.118,30.752)$ & 0.000 & $31.212(11.024,51.400)$ & 0.002 \\
\hline \multicolumn{5}{|l|}{ Discharge to: } \\
\hline Own home & referent & & referent & \\
\hline Other & $-2.279(-6.918,2.360)$ & 0.336 & $4.196(-5.187,13.579)$ & 0.381 \\
\hline \multicolumn{5}{|l|}{ Fracture type: } \\
\hline Intertrochanteric & referent & & referent & \\
\hline Intracapsular, displaced & $6.495(-.570,13.561)$ & 0.072 & $-1.072(-15.117,12.974)$ & 0.881 \\
\hline Intracapsular, undisplaced & $-1.650(-8.956,5.657)$ & 0.658 & $5.774(-9.135,20.684)$ & 0.448 \\
\hline Subtrochanteric & $3.412(-.437,7.262)$ & 0.082 & $0.472(-6.980,7.924)$ & 0.901 \\
\hline \multicolumn{5}{|l|}{ Operation: } \\
\hline Other & referent & & referent & \\
\hline Total hip replacement & $-2.245(-8.885,4.395)$ & 0.508 & $5.575(-19.021,7.870)$ & 0.416 \\
\hline
\end{tabular}

2 or less for analysis, as this score is largely subjective, with small numbers at the extremes of the grading system in our sample, and the fact that some cases were graded as ASA grade 1, which is unusual for hip fractures. Owing to the unavailability of the data, postoperative complications could not be analysed in our sample; however, higher ASA gradings are associated with an increased rate of postoperative complications, ${ }^{22}$ as well as increased length of stay. ${ }^{23}$ 
They are also associated with delay to theatre and higher mortality rates. ${ }^{23}$ In-hospital mortality is also affected by nurse to patient ratio, with one study showing a $16 \%$ increase in in-hospital mortality rates for each decrease in full-time equivalent registered nurse per patient day. ${ }^{24} \mathrm{~A}$ multidisciplinary approach is vital from a range of services to ensure that these patients are optimised both prior to surgery and postoperatively to decrease complication rates and optimise outcomes. ${ }^{25}$

There was no statistically significant difference in length of stay between male and female patients in our cohort. There may be a confounding element of selection bias in this finding as males have higher rates of mortality and institutionalization $;^{26}$ however, this finding is consistent with other literature on the subject. ${ }^{27}$ Previous studies have shown higher mortality and increased complications in males with hip fractures. ${ }^{28}$ Males are often younger and have more comorbidities at time of hip fracture. ${ }^{29,30}$ While gender may not influence length of stay, it is important to consider these comorbidities and increased mortality when caring for male patients with hip fracture.

We found no difference in length of stay with different fracture patterns of which $37.3 \%$ were intertrochanteric, $49.7 \%$ were displaced and $6.7 \%$ undisplaced intracapsular, and $6.4 \%$ were subtrochanteric. This mirrors similar observational studies. ${ }^{9}$ There are some studies which show an increased length of stay with intertrochanteric and subtrochanteric fractures. ${ }^{10,31}$ This association may be confounded by weightbearing restrictions in some units; however, in line with national guidance all hip fractures are operated on with the aim of allowing full weightbearing in our unit. ${ }^{17}$ We suggest that the factors of each individual patient are more important in determining length of stay rather than the fracture itself.

Patients undergoing THR had a significantly shorter length of stay than those undergoing other procedures. Length of stay did not reach statistical significance as an independent factor, however. This may be because the decision to perform a THR rather than hemiarthroplasty is based on good mobility, absence of cognitive impairment and anaesthetic fitness, as per UK national guidelines. ${ }^{17}$ These factors were shown to be independent predictors of shorter length of stay and it is likely that the patient selection rather than the operation itself is responsible for this difference. Patients with these factors are likely to be discharged early and to their own homes. Data from the UK National Joint Registry shows outcomes of THR for hip fractures are similar to those when performed for other indications such as osteoarthritis. ${ }^{32}$ A large meta-analysis has shown an increased dislocation rate when compared with hemiarthroplasty. ${ }^{33}$ This and the greater operative risks may discourage surgeons from performing THR, which is only currently carried out on $32 \%$ of patients in the UK who are deemed suitable by national guidelines. ${ }^{34}$

Whether patients were admitted from their own home or other care facility did not have an independent effect on the length of stay in our study. This differs from an Australian study, where length of stay was considerably longer in patients admitted from their own home. ${ }^{29}$ The same is true for the location to which patients are discharged. Discharge to a new nursing or residential home placement does, however, have a large independent effect on the total length of stay. It is important to note that any change in patients' residence and discharge destination require careful consideration and planning and will prolong total healthcare stay.

We found a statistically significant difference in length of stay with age in patients during the unadjusted analysis. In our regression model, age was not shown to be a statistically significant independent factor. This suggests that the associated increased rates of comorbidities, dementia and decreased mobility associated with ageing are responsible for the difference rather than age being an independent factor. Hence, the management of patients and rehabilitation decisions should not be based on age but on other associated predictive factors. Studies have shown increased short-term mortality rates with increasing age; however, one-year mortality is similar to that of the background population. ${ }^{35}$

Non-patient factors were not measured in our study and are more difficult to quantify in general. They are however vitally important in governing patients' length of stay and explain the large variation in length of stay throughout the UK, as well as worldwide, despite similar patient demographics.

The British Orthopaedics Association and British Geriatrics Society's blue book emphasises the importance of the role of an orthogeriatrician in ensuring best outcomes in fragility fractures. $^{25}$ Orthogeriatrician input minimises delay to surgery, reduces complications and facilitates better multidisciplinary care. ${ }^{25}$ While the input of an orthogeriatrician is recommended in national guidelines, ${ }^{17}$ the way the service is delivered varies considerably within the UK. A financial incentive, the Best Practice Tariff, is awarded to hospitals in England if patients are managed under shared orthopaedic and geriatric care, reviewed by a senior orthogeriatrician within 72 hours and have a geriatrician-led multidisciplinary rehabilitation along with six other criteria. $^{36}$

There was no significant difference in length of stay in patients assessed preoperatively by an orthogeriatrician, neither was there a correlation between time to first orthogeriatric assessment and length of stay in our patient group. There may well be a reduction in surgery delays and postoperative complications, which were not measured in our population. The introduction of a geriatrician-led care model to one district general hospital in South Wales led to an increase in rates of surgery within 48 hours, as well as a reduction in total length of stay from 32.0 to 19.6 days when compared with a weekly orthogeriatrician review. ${ }^{35}$ These once-weekly reviews have been shown to have no effect on patient mortality, length of stay or discharge placement. ${ }^{37} \mathrm{~A}$ daily geriatric collaborative model has shown a $21 \%$ reduction in medical complications, a $3 \%$ reduction in mortality and a $20 \%$ reduction in readmissions at six months. ${ }^{38} \mathrm{~A}$ Spanish study reported that an orthogeriatric unit saved up to $€ 3,741$ per patient in cost. ${ }^{39}$ Our study was undertaken in a hospital with an 
orthopaedic-led care with twice weekly orthogeriatrician presence. This may explain the difference in acute and total length of stay when compared with the UK national average.

There was no correlation between time from admission to surgery and acute or total length of stay in our dataset. Studies have shown a decrease in mortality, complications and length of stay with a delay to surgery. ${ }^{40}$ In our unit, patients with hip fractures are prioritised for early surgery, as in most centres in the UK, and therefore the time to surgery was generally low, with a median time from arrival in the emergency department to surgery of 23 hours.

Rehabilitation in intermediate care facilities is also a key component of hip fracture management. Its availability varies throughout the UK in terms of waiting times and availability of home-based community rehabilitation services. ${ }^{41}$ These variations will clearly affect both acute and total length of stay. The input of allied health services such as physiotherapy, occupational therapy, social workers and dietetics, all lead to better service for patients to improve outcomes, including length of stay. The coordination of these services is best provided by an orthogeriatrician. ${ }^{25}$

Despite the limitations of a single-centre retrospective study, we have been able to identify and quantify the effect of independent patient factors affecting length of stay. By assessing these factors early, this information can be used to plan services and attempt to reduce their effect to optimise outcomes. This should occur in conjunction with the national standard of multidisciplinary orthogeriatrician-led approach and improvements in availability of rehabilitation and allied services. If these steps are followed, substantial cost savings can be made, as well as improved patient satisfaction and better availability of acute and rehabilitation beds.

\section{Conclusions}

Low AMTS and pre-fracture mobility were found to be independent factors affecting acute hospital and total health service length of stay, as well as ASA grade for acute hospital length of stay in our patient cohort.

\section{References}

1. Leal J. Impact of hip fracture on hospital care costs: a population based study. Osteoporos Int. 2016; 27: 549-558.

2. Burge RT, Worley D, Johansen A et al. The cost of osteoporotic fractures in the United Kingdom. J Med Econ 2001; 4(1-4): 51-62.

3. Medin E, Goude F, Melberg $\mathrm{HO}$ et al. European regional differences in all-cause mortality and length of stay for patients with hip fracture. 2015; 24(Suppl 2): 53-64.

4. Thakore RV, Foxx AM, Lang MF et al. Operative intervention for geriatric hip fracture: does type of surgery affect hospital length of stay? Am J Orthop (Belle Mead NJ) 2015; 44(5): 228-232.

5. Nikkel L, Kates SL, Schreck M et al. Length of hospital stay after hip fracture and risk of early mortality after discharge in New York state: retrospective cohort study. BMJ 2015; 351: h6,246.

6. Nordström P, Gustafson Y, Michaëlsson K, Nordström A. Length of hospital stay after hip fracture and short term risk of death after discharge: a total cohort study in Sweden. BMJ 2015; 350; h696.

7. Brainsky A, Glick H, Lydick $E$ et al. The economic cost of hip fractures in community-dwelling older adults: a prospective study. J Am Geriatr Soc 1997; 45(3): 281-287.
8. Chen LT. Hip fractures in the elderly: impact of comorbid illnesses on hospitalisation costs. Ann Acad Med Singapore 2007; 36(9): 784-787.

9. Seitz DP, Adunuri N, Gill SS, Rochon PA. Prevalence of dementia and cognitive impairment among older adults with hip fractures. J Am Med Dir Assoc 2011; 12: $556-564$.

10. Wehren LE, Hawkes WG, Orwig DL et al. Gender differences in mortality after hip fracture: the role of infection. J Bone Miner Res 2003; 18: 2,231-2,237.

11. Handoll HH, Farrar MJ, McBirnie J et al. Heparin, low molecular weight heparin and physical methods for preventing deep vein thrombosis and pulmonary embolism following surgery for hip fractures. Cochrane Database Syst Rev 2002; (4): CD000305.

12. Clague JE Craddock E, Andrew $G$ et al. Predictors of outcome following hip fracture.Admission time predicts length of stay and in-hospital mortality. Injury 2002; 33(1): 1-6.

13. Li L, Ren J, Liu J. What are the risk factors for dislocation of hip bipolar hemiarthroplasty through the anterolateral approach? A nested case-control study. Clin Orthop Relat Res 2016; 474(12): 2,622-2,629.

14. Muir S, Yohannes AM. The impact of cognitive impairment on rehabilitation outcomes in elderly patients admitted with a femoral neck fracture: a systematic review. J Geriatr Phys Ther 2009; 32(1): 24-32.

15. Holt E, Evans RA, Hindley CJ, Metcalfe JW. 1000 femoral neck fractures: the effect of pre-injury mobility and surgical experience on outcome. Injury 1994; 25(2): 91-95.

16. Yeoh C, Fazal MA. ASA Grade and elderly patients with femoral neck fracture. Geriatr Orthop Surg Rehabil 2014; 5(4): 195-199.

17. Kastanis G, Topalidou A, Alpantaki $\mathrm{K}$ et al. Is the ASA score in geriatric hip fractures a predictive factor for complications and readmission? Scientifica (Cairo) 2016; 2016: 7096245.

18. Kannegaard PN, van der Mark S, Eiken P, Abrahamsen B. Excess mortality in men compared with women following a hip fracture. National analysis of comedications, comorbidity and survival. Age Ageing 2010; 39(2):203-209.

19. Dudkiewicz I, Burg A, Salai M, Hershkovitz A. Gender differences among patients with proximal femur fractures during rehabilitation. Gend Med 2011; 8 (4): 231-238.

20. Mangram A, Moeser P, Corneille MG et al. Geriatric trauma hip fractures: is there a difference in outcomes based on fracture patterns? World J Emerg Surg 2014; 9: 59.

21. Ireland A, Kelly PJ, Cumming RG. Total hospital stay for hip fracture: measuring the variations due to pre-fracture residence, rehabilitation, complications and comorbidities. BMC Health Serv Res 2015; 15: 17

22. Sund R, Riihimäki J, Mäkelä $M$ et al. Modeling the length of the care episode after hip fracture: does the type of fracture matter? Scand J Surg 2009; 98(3): 169-174.

23. National Institute for Health and Clinical Excellence. Hip Fracture: Management. Clinical Guideline CG124. London: NICE; 2011 (last updated May 2017).

24. Stafford GH, Charman SC, Borroff MJ et al. Total hip replacement for the treatment of acute femoral neck fractures: results from the National Joint Registry of England and Wales at 3-5 years after surgery. Ann R Coll Surg Engl 2012; 94(3): 193-198.

25. Hopley C, Stengel D, Ekkernkamp A, Wich M. Primary total hip arthroplasty versus hemiarthroplasty for displaced intracapsular hip fractures in older patients: systematic review. BMJ 2010; 340: c2,332.

26. Brown CA, Olson $S$, Zura R. Predictors of length of hospital stay in elderly hip fracture patients. J Surg Orthop Adv 2013; 22(2): 160-163.

27. Liebermann $D$, Lieberman $D$. Rehabilitation following hip fracture surgery: a comparative study of females and males. Disabil Rehabil 2004; 26(2): 85-90.

28. Sterling R. Gender and race/ethnicity differences in hip fracture incidence, morbidity, mortality, and function. Clin Orthop Relat Res 2011; 469(7): 1,913-1,918.

29. Holt G, Macdonald D, Fraser M, Reece AT. Outcome after surgery for fracture of the hip in patients aged over 95 years. J Bone Joint Surg Br 2006; 88(8): 1,060-1,064.

30. Monitor; NHS England. 2014/15 National Tariff Payment System. London: Monitor; 2015.

31. Neuburger J, Harding KA, Bradley RJ et al. Variation in access to community rehabilitation services and length of stay in hospital following a hip fracture: a cross-sectional study. BMJ Open 2014; 4(9): e005469.

32. Gupta A. The effectiveness of geriatrician-led comprehensive hip fracture collaborative care in a new acute hip unit based in a general hospital setting in the UK. J R Coll Physicians Edinb 2014; 44(1): 20-26.

33. Gilchrist WJ, Newman RJ, Hamblen DL, Williams BO. Prospective randomised study of a orthopaedic geriatric in patient service. BMJ 1988; 297: 1,116-1,118. 
34. Choudry H. Total hip arthroplasty after hip fracture. BMJ 2016; 353: i2,217.

35. Fisher AA, Davis MW, Rubenach SE et al. Outcomes for older patients with hip fractures: the impact of orthopaedic and geriatric medicine cocare. J Orthop Trauma 2006; 20: 172-180.

36. González Montalvo JI, Gotor Pérez P, Martín Vega A et al. The acute orthogeriatric unit: assessment of its effect on the clinical course of patients with hip fractures and an estimate of its financial impact. Rev Esp Geriatr Gerontol 2011; 46: 193-199.

37. Grigoryan K, Javedan H, Rudolph JL. Orthogeriatric care models and outcomes in hip fracture patients: a systematic review and meta-analysis. J Orthop Trauma 2014; 28(3): e49-e55.

38. Burgers P, Van Geene AR, Van den Bekerom MP et al. Total hip arthroplasty versus hemiarthroplasty for displaced femoral neck fractures in the healthy elderly: a meta-analysis and systematic review of randomized trials. Int Orthop 2012; 36: 1,549-1,560.

39. Royal Australian College of General Practitioners. Medical Care of Older Persons in Residential Aged Care Facilities (Silver Book). East Melbourne: RACGP; 2006.
40. National Hip Fracture Database. Annual Report 2016. London: Royal College of Physicians; 2016.

41. British Orthopaedic Association; British Geriatrics Society. The Care of Patients with Fragility Fracture ('Blue Book'). London: BGS; 2007.

42. Burge R, Dawson-Hughes B, Solomon DH et al. Incidence and economic burden of osteoporosis-related fractures in the United States, 2005-2025. J Bone Miner Res 2007; 22: 465-475.

43. Handoll H, Sherrington C, Mak JC. Interventions for improving mobility after hip fracture surgery in adults (Review). Cochrane Database Syst Rev 2011; (3): CD001704.

44. Bachmann S, Finger C, Huss A et al. Inpatient rehabilitation specifically designed for geriatric patients: systematic review and meta-analysis of randomised controlled trials. BMJ 2010; 340: c1,718.

45. Schilling P, Goulet JA, Dougherty PJ. Do higher hospital-wide nurse staffing levels reduce in-hospital mortality in elderly patients with hip fractures: a pilot study. Clin Orthop Relat Res 2011; 469(10): 2,932-2,940.

46. Fransen $\mathrm{M}$, Woodward $\mathrm{M}$, Norton $\mathrm{R}$ et al. Excess mortality or institutionalization after hip fracture: men are at greater risk than women. J Am Geriatr Soc 2002; 50: 685-690. 